Volume 135, Number 12, December 2007, Pages 4023-4029

S 0002-9939(07)09265-9

Article electronically published on September 5, 2007

\title{
DEGREE ONE MAPS BETWEEN HYPERBOLIC SMALL 3-MANIFOLDS
}

\author{
MICHEL BOILEAU AND SHICHENG WANG
}

(Communicated by Daniel Ruberman)

\begin{abstract}
We construct the first examples of degree one maps between nonhomeomorphic closed hyperbolic small 3-manifolds.
\end{abstract}

\section{INTRODUCTION}

All terminologies not defined in this paper are standard; see [Ja], [Ro] and [Th]. A compact 3-manifold $M$ is small if it is orientable, irreducible and if any incompressible surface in $M$ is parallel to $\partial M$. A knot $k$ in a 3-manifold $M$ is small if its exterior $M-\operatorname{int} \mathcal{N}(k)$, denoted by $E(k)$, is a small 3 -manifold.

The main theme of this work is to study the existence of degree one maps between closed hyperbolic small 3-manifolds. All known and expected closed small 3-manifolds are either Seifert fibered or hyperbolic. The existence of degree one maps between closed small Seifert manifolds has been studied (see [HWZ] and the references there).

For closed hyperbolic small 3-manifolds, even some general results for degree one maps between them have been established (see [RW] and [BW2]), but to our knowledge, there are no known examples of such degree one maps. Indeed the authors of $[R W]$ wondered how to find such degree one maps when they were working on [RW], which is the first motivation of the present work.

Note that there are many ways of producing degree one maps between closed hyperbolic 3-manifolds (cf. [BW1], [Ka], [Ru]), but none of them insure that both hyperbolic manifolds are small. The following theorem provides the first examples of degree one maps between non-homeomorphic closed small hyperbolic 3-manifolds.

Theorem 1.1. There are infinitely many pairs of non-homeomorphic, closed, small hyperbolic 3-manifolds $(M, N)$ such that there is a degree one map $f: M \rightarrow N$.

Remark 1.2. The construction of degree one maps in Theorem 1.1 is based on Thurston's hyperbolic Dehn surgery theorem [Th] and Proposition 2.2, which provides non-trivial proper degree one maps between the exteriors of hyperbolic small knots in the 3 -sphere. These are also, to our knowledge, the first such examples. Our examples of degree one maps in Proposition 2.2 are constructed from the exteriors of some $n$-bridge knots, with $n>2$, to the exteriors of some 2-bridge knots.

Received by the editors March 16, 2006 and, in revised form, June 21, 2006.

2000 Mathematics Subject Classification. Primary 57M50.

The second author is partially supported by MSTC and NSFC. 
It would be interesting to find a degree one map between the exteriors of two hyperbolic 2-bridge knots, ${ }^{1}$ since such a degree one map will produce degree one maps between closed 3-manifolds obtained by Dehn surgeries on those knots with the same coefficients, and for most given coefficients those two closed 3-manifolds are small and hyperbolic.

In general we wonder if there is a degree one map $f: E\left(k_{1}\right) \rightarrow E\left(k_{2}\right)$ for $k_{1}$ and $k_{2}$ two knots in $S^{3}$, is the bridge number of $k_{1}$ not smaller than that of $k_{2}[\mathrm{~W}]$ ?

\section{EXAMPles of DEGREe ONE MAPS BETWEEN SMALL HYPERBOLIC 3-MANIFOLDS}

This section is devoted to the proof of Theorem 1.1. Our construction uses tangle sum in the sense of Conway.

A tangle $T=\left(B^{3}, a_{1} \cup a_{2}\right)$ is a properly embedded disjoint pair of $\operatorname{arcs}\left(a_{1} \cup\right.$ $\left.a_{2}, \partial a_{1} \cup \partial a_{2}\right) \hookrightarrow\left(B^{3}, \partial B^{3}\right)$.

Such a tangle is irreducible if there is no 2-sphere $S \subset B^{3}$ meeting transversely an $\operatorname{arc} a_{i}$ in two points such that the intersection of the 3-ball $V$ bounded by $S$ in $B^{3}$ and $a_{i}$ is a knotted arc in $V$.

We denote by $T_{0}=\left(B^{3}, b_{1} \cup b_{2}\right)$ the trivial tangle. It is formed by two unknotted arcs separated by a properly embedded disk in $B^{3}$ disjoint from them.

Our construction of non-trivial degree one maps between small closed hyperbolic 3-manifolds relies on Thurston' hyperbolic Dehn surgery theorem and the following two propositions:

Proposition 2.1. There exists an irreducible, non-trivial tangle $T=\left(B^{3}, a_{1} \cup a_{2}\right)$ with the following properties:

(1) The 2-fold covering of $B^{3}$ branched along $a_{1} \cup a_{2}$ is the exterior $E$ of a small hyperbolic knot in $S^{3}$.

(2) There is a proper degree one map $f: T=\left(B^{3}, a_{1} \cup a_{2}\right) \rightarrow T_{0}=\left(B^{3}, b_{1} \cup b_{2}\right)$ onto the trivial tangle $T_{0}$ such that

- the restriction $\left.f\right|_{\partial B^{3}}: \partial B^{3} \rightarrow \partial B^{3}$ is a homeomorphism,

- for $i \in\{1,2\}, f^{-1}\left(b_{i}\right)=a_{i}$ and the restriction $f \mid: a_{i} \rightarrow b_{i}$ is a homeomorphism.

By using a Conway sum of the tangle $T$ with rational tangles, we deduce from Proposition 2.1 the following result:

Proposition 2.2. There are infinitely many hyperbolic small knots in $S^{3}$ with bridge number $\geq 3$ such that their exteriors admit a proper degree one map to the exterior of a hyperbolic 2-bridge knot.

The three subsections below are devoted to the proofs of Proposition 2.1, Proposition 2.2 and Theorem 1.1, respectively.

2.1. Construction of the tangle $T$ and proof of Proposition 2.1. We consider the non-alternating knot $\tilde{k}$ with eight crossings, the knot $8_{21}$ in Rolfsen's book tabulation [Ro]. It is the Montesinos knot $\mathcal{M}(1 ; 1 / 2,2 / 3,2 / 3)$ with 3 -branches in the notation of $[\mathrm{BoZ}]$. By Oertel's work [O1] it is a small hyperbolic knot. It is also a fibred knot with fibre a surface of genus 2; see for example [Ga].

\footnotetext{
${ }^{1}$ After the acceptance of this paper, T. Ohtsuki, R. Riley and M. Sakuma [ORS] informed us that they have constructed degree one maps between 2-bridge knot complements.
} 


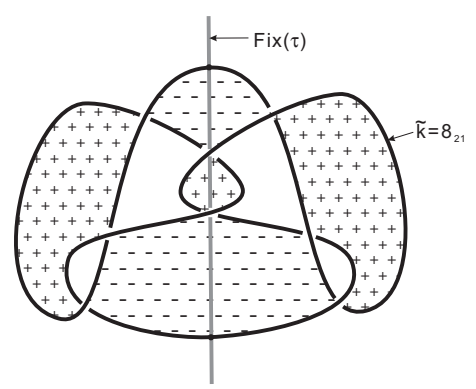

Figure 1

Moreover, it is a strongly invertible knot; i.e., there is a smooth involution $\tau$ of the pair $\left(S^{3}, \tilde{k}\right)$ such that $F i x(\tau)$, the fixed point set of $\tau$, is an unknotted circle and meets $\tilde{k}$ in exactly two points (Figure 1 ).

After an isotopy of the fibration of the exterior $E=E(\tilde{k})$, we can assume that the restriction of $\tau$ on $E$ is fibre preserving. Hence there are two fibres $F_{1}$ and $F_{2}$ invariant by $\tau$ in $E$. Moreover $\operatorname{Fix}(\tau) \cap E=\tilde{a}_{1} \cup \tilde{a}_{2}$, where $\tilde{a}_{i}$ is a properly embedded arc in $F_{i}$ which separates $F_{i}$ into two symmetric parts, $i=1,2$ (Figure $1)$.

The orbifold quotient $E / \tau$ has an underlying space $|E / \tau|$ homeomorphic to $B^{3}$ and the ramification locus formed by the union of two properly embedded disjoint $\operatorname{arcs} a_{1} \cup a_{2}$ in $E / \tau$. That gives naturally a tangle $T=\left(|E / \tau|, a_{1} \cup a_{2}\right)$, which by construction verifies property (1) of Proposition 2.1.

To verify property (2), we construct a proper degree one map $\tilde{f}: E \rightarrow S^{1} \times D^{2}$, which is equivariant with respect to the involution $\tau$ on $E$ and to the involution $\tau_{0}$ obtained on $S^{1} \times D^{2}$ by extending the involution $\left.\tau\right|_{\partial E}$ to the solid torus.

We identify $\partial E$ with $S^{1} \times \partial D^{2}$ by choosing a preferred meridian-longitude coordinate system $(\tilde{\mu}, \tilde{\lambda})$ on $\partial E$ and by identifying the meridian $\tilde{\mu}$ with $S^{1} \times\{\star\}$ and the longitude $\tilde{\lambda}$, which is the boundary of a fibre of the fibration of $E$, with $\{\star\} \times \partial D^{2}$.

The involution $\tau_{0}$ preserves two meridian disks $D_{1}$ and $D_{2}$, and Fix $\left(\tau_{0}\right) \cap D_{i}=\tilde{b}_{i}$ is a properly embedded arc in $D_{i}$ for $i \in\{1,2\}$. In particular the orbifold quotient $S^{1} \times D^{2} / \tau_{0}$ has $B^{3}$ as its underlying space and the union of the two disjoint properly embedded arcs $b_{1} \cup b_{2}$ as its ramification locus. That gives a trivial tangle $T_{0}=$ $\left(\left|S^{1} \times D^{2} / \tau_{0}\right|, b_{1} \cup b_{2}\right)$.

The construction of the equivariant degree one map $\tilde{f}$ is done in three steps:

Step 1. By the choice of $\tau_{0}$ on $S^{1} \times D^{2}$ and the identification of $\partial E$ with $S^{1} \times \partial D^{2}$, we can take $\tilde{f}: \partial E \rightarrow \partial\left(S^{1} \times D^{2}\right)$ to be the identity.

Step 2. We extend $\tilde{f}$ equivariantly to the two fibres $F_{1}$ and $F_{2}$, so that for $i \in\{1,2\}, \tilde{f}\left(F_{i}\right)=D_{i}, \tilde{f}^{-1}\left(\tilde{b}_{i}\right)=\tilde{a}_{i}$ and $\left.\tilde{f}\right|_{\tilde{a}_{i}}: \tilde{a}_{i} \rightarrow \tilde{b}_{i}$ is a homeomorphism.

Since the properly embedded $\operatorname{arc} \tilde{a}_{i} \subset F_{i}$ is separating, we have $F_{i}=F_{i}^{\prime} \cup \tau\left(F_{i}^{\prime}\right)$ for $i \in\{1,2\}$, where $F_{i}^{\prime}$ is a genus 1 surface. In the same way, we have $D_{i}=$ $D_{i}^{\prime} \cup \tau\left(D_{i}^{\prime}\right)$ for $i \in\{1,2\}$, where $D_{i}^{\prime}$ is a disk.

We consider the pinch $p_{i}: F_{i}^{\prime} \rightarrow D_{i}^{\prime}$ which is the identity on a collar neighborhood of $\partial F_{i}^{\prime}$, for $i \in\{1,2\}$. Then we extend $\tilde{f}$ equivariantly on $F_{i}$ by taking $\left.\tilde{f}\right|_{F_{i}^{\prime}}=p_{i}$ and $\left.\tilde{f}\right|_{\tau\left(F_{i}^{\prime}\right)}=\tau_{0} \circ p_{i} \circ \tau$, for $i \in\{1,2\}$. 


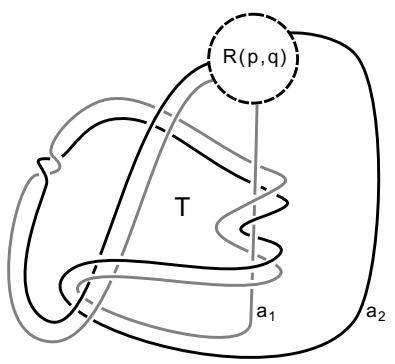

FiguRE 2

At this point, for $i \in\{1,2\}, \tilde{f}\left(F_{i}\right)=D_{i}, \tilde{f}^{-1}\left(\tilde{b}_{i}\right)=\tilde{a}_{i}$ and $\tilde{f} \mid: \tilde{a}_{i} \rightarrow \tilde{b}_{i}$ is a homeomorphism.

Step 3. We extend $\tilde{f}$ equivariantly to the remaining part of $E$.

By cutting $E$ along the two fibres $F_{1} \cup F_{2}$, we get $E=F \times[1,2] \cup \cup_{F_{1} \cup F_{2}} \tau(F \times[1,2])$. In the same way $S^{1} \times D^{2}=D^{2} \times[1,2] \cup_{D_{1} \cup D_{2}} \tau_{0}\left(D^{2} \times[1,2]\right)$.

From Steps 1 and 2 we have defined a degree one map $\tilde{f}: \partial(F \times[1,2]) \rightarrow$ $\partial\left(D^{2} \times[1,2]\right)$. Since $D^{2} \times[1,2]$ is a 3 -ball, we can extend this map to a degree one $\operatorname{map} \tilde{f}: F \times[1,2] \rightarrow D^{2} \times[1,2]$.

Since $\tilde{f}: F_{1} \cup F_{2} \cup \partial E \rightarrow D_{1} \cup D_{2} \cup \partial\left(S^{1} \times D^{2}\right)$ is already equivariant with respect to $\tau$ and $\tau_{0}$, we can finally define the map $\tau_{0} \circ \tilde{f} \circ \tau: \tau(F \times[1,2]) \rightarrow \tau_{0}\left(D^{2} \times[1,2]\right)$ to get the desired proper degree one map $\tilde{f}: E \rightarrow S^{1} \times D^{2}$ with the following properties:

(a) $\tau_{0} \circ \tilde{f}=\tilde{f} \circ \tau$.

(b) The restriction $\left.\tilde{f}\right|_{\partial E}: \partial E \rightarrow S^{1} \times \partial D^{2}$ is the identity with respect to the chosen parametrization of $\partial E$.

(c) For $i \in\{1,2\}, \tilde{f}^{-1}\left(\tilde{b}_{i}\right)=\tilde{a}_{i}$ and the restriction $\left.\tilde{f}\right|_{\tilde{a}_{i}}: \tilde{a}_{i} \rightarrow \tilde{b}_{i}$ is a homeomorphism.

Now this equivariant proper degree one map $\tilde{f}$ covers through the involutions $\tau$ and $\tau_{0}$ a proper degree one map: $f: T=\left(|E / \tau|, a_{1} \cup a_{2}\right) \rightarrow T_{0}=\left(\left|S^{1} \times D^{2} / \tau_{0}\right|, b_{1} \cup\right.$ $b_{2}$ ), between the tangle $T$ and the trivial tangle $T_{0}$. This degree one map verifies the properties of (2) of Proposition 2.1 because of properties (b) and (c) of $\tilde{f}$. That finishes the proof of Proposition 2.1.

2.2. Proof of Proposition 2.2. In the parametrization of $\partial E$ by the preferred meridian-longitude pair $(\tilde{\mu}, \tilde{\lambda})$, any simple closed curve on $\partial E$ is determined by a unique slope $(p, q)$, where $p \geq 0$ and $q \in \mathbb{Z}$ are coprime. We denote by $E(p, q)$ the closed orientable 3 -manifold obtained from $E$ by Dehn filling $\partial E$ along the slope $(p, q)$.

The involution $\tau$ on the knot exterior $E$ can be extended to the glued solid torus to get an involution still called $\tau$ on the closed 3-manifold $E(p, q)$. According to Montesinos's construction [Mo], the quotient of $E(p, q)$ by $\tau$ is $S^{3}$ and the branching locus $k(p, q)$ is a knot or a link with two components, according to whether $p$ is odd or even.

This knot or link $k(p, q)$ is obtained by a Conway sum of the tangle $T$ with the rational tangle $R(p, q)$ of type $(p, q)$ (see Figure 2 ), where the outside tangle $T$ is drawn by using Montesinos's technique. 
Now, the degree one map $f: T \rightarrow T_{0}$ can be extended trivially by a homeomorphism to a degree one map of pairs $g:\left(S^{3}, k(p, q)\right) \rightarrow\left(S^{3}, b(p, q)\right)$, such that

- $b(p, q)$ is the 2-bridge knot or link, obtained by a Conway sum of the trivial tangle $T_{0}$ with the rational tangle $R(p, q)$,

- $g^{-1}(b(p, q))=k(p, q)$ and the restriction $\bar{g} \mid: k(p, q) \rightarrow b(p, q)$ is a homeomorphism.

This last property follows immediately from the properties of (2) of $f$ in Proposition 2.1 .

By considering the restriction of $g$ to the exterior of $k(p, q)$, Proposition 2.2 follows now from the following lemma:

Lemma 2.3. For $p=2 p^{\prime}+1, p^{\prime}>1$ and $q \neq n p \pm 1, n \in \mathbb{Z}, k(p, q)$ and $b(p, q)$ are small hyperbolic knots in $S^{3}$. Moreover $k(p, q)$ has bridge number $\geq 3$.

Proof. By the classification of 2-bridge knots or links (cf. [BuZ]), $b(p, q)$ is a hyperbolic knot iff $p=2 p^{\prime}+1, p^{\prime}>1$ and $q \neq n p \pm 1, n \in \mathbb{Z}$. By [HT] it is a small knot.

By Oertel [O2] (see also [Dun1]) the boundary slopes of the knot $8_{21}$ are the following integral slopes:

$$
\{(12,-1),(6,-1),(2,-1),(0,1),(1,1)\} .
$$

In particular all odd $p>1$ are not in this list. So for the slopes $(p, q)$ given in Lemma 2.3, the closed 3-manifolds $E(p, q)$ are small.

Since $E(p, q)$ is the 2-fold branched covering of the knot $k(p, q)$, it follows from the equivariant Dehn lemma that $k(p, q)$ is a small knot in $S^{3}$ (cf. [GL]). Hence it is either a hyperbolic or a torus knot.

It cannot be a torus knot since its exterior admits a proper degree one map onto the exterior of a hyperbolic 2-bridge knot. This would contradict the fact that the simplicial volume of a torus knot exterior vanishes, while it is always non-zero for a hyperbolic knot exterior.

The knot $k(p, q)$ has bridge number $\geq 3$. Otherwise its 2 -fold branched covering would be a lens space and by the cyclic surgery theorem [CGLS] $q$ would be equal to \pm 1 , since $\tilde{k}$ is a hyperbolic knot. This contradicts our choice for $q$.

That finishes the proof of Lemma 2.3 and hence of Proposition 2.2.

2.3. Degree one map between closed small hyperbolic 3-manifolds and proof of Theorem 1.1. In Proposition 2.2 we constructed a small hyperbolic knot $k_{1} \subset S^{3}$ with bridge number $\geq 3$ and a hyperbolic 2-bridge knot $k_{2} \subset S^{3}$ such that there is a degree one map $g:\left(S^{3}, k_{1}\right) \rightarrow\left(S^{3}, k_{2}\right)$ such that $g^{-1}\left(k_{2}\right)=k_{1}$ and such that the restriction $\bar{g} \mid: k_{1} \rightarrow k_{2}$ is a homeomorphism. Let $E_{1}$ and $E_{2}$ be the exteriors of $k_{1}$ and $k_{2}$, respectively. As before we choose for $i \in\{1,2\}$ a trivialization of $\partial E_{i}$ by a preferred meridian-longitude pair $\left(\mu_{i}, \lambda_{i}\right)$. Then (after possibly some isotopy on the boundary) $g$ induces a proper degree one map $h: E_{1} \rightarrow E_{2}$ such that

- the restriction $h \mid: \partial E_{1} \rightarrow \partial E_{2}$ is a homeomorphism,

- $h\left(\mu_{1}\right)=\mu_{2}$ and $h\left(\lambda_{1}\right)=\lambda_{2}$.

For any slope $(r, s)$ on $\partial E_{i}, i=1,2$, this degree one map $h$ extends trivially by a homeomorphism to a degree one map $h_{r, s}: E_{1}(r, s) \rightarrow E_{2}(r, s)$. Now Theorem 1.1 is a consequence of the following lemma: 
Lemma 2.4. For almost all slopes $(r, s)$ (i.e. except finitely many), the two closed orientable 3-manifolds $E_{1}(r, s)$ and $E_{2}(r, s)$ are small, hyperbolic and not homeomorphic.

Proof. By [Hat] there are only finitely many slopes $(p, q)$ that can be boundary slopes on either $\partial E_{1}$ or $\partial E_{2}$. Since $k_{1}$ and $k_{2}$ are small knots in $S^{3}$, if $(r, s)$ avoids this finite set of slopes, then $E_{1}(r, s)$ and $E_{2}(r, s)$ are small, closed 3-manifolds.

Let $v_{i}=\operatorname{vol}\left(E_{i}\right)$ be the hyperbolic volume of $E_{i}, i \in\{1,2\}$. Since $k_{1}$ is not a 2-bridge knot, $E_{1}$ is not homeomorphic to $E_{2}$, because knots are determined by their complement in $S^{3}$. Since there is a proper degree one $h: E_{1} \rightarrow E_{2}$, GromovThurston's strict rigidity theorem ([Th, Chap. 6], [Dun2]) implies that $v_{1}>v_{2}$.

By Thurston's hyperbolic Dehn surgery theorem ([Th, Chap. 4]; see also [BP, Appendix B]) and Schläfli's formula (cf. [Mi]), there is a constant $c>0$ (depending only on $k_{1}$ and $k_{2}$ ) such that for $r^{2}+s^{2} \geq c^{2}$ the following happens:

- both $E_{1}(r, s)$ and $E_{2}(r, s)$ are hyperbolic,

- $v_{1}>\operatorname{vol}\left(E_{1}(r, s)\right)>v_{2}>\operatorname{vol}\left(E_{2}(r, s)\right)$.

Therefore if $(r, s)$ avoids the finite set of slopes $r^{2}+s^{2}<c^{2}$, then $E_{1}(r, s)$ and $E_{2}(r, s)$ are both hyperbolic and not mutually homeomorphic.

This finishes the proof of Lemma 2.4 and of Theorem 1.1.

Remark 2.5. With further effort, one can show that the bridge number of the knots $k(p, q)$ in Proposition 2.2 is at most 4 and the Heegaard genus of the 3-manifolds $E_{1}(r, s)$ in Lemma 2.3 is at most 3.

\section{ACKNOWLEDGMENTS}

We wish to thank A. Kawauchi and A. Reid for helpful conversations.

\section{REFERENCES}

[BP] M. Boileau and J. Porti, Geometrization of 3-orbifolds of cyclic type, Astérisque Monograph, 272, 2001. MR1844891 (2002f:57034)

[BW1] M. Boileau and S.C. Wang, Non-zero degree maps and surface bundles over $S^{1}$, J. Diff. Geom. 43, 1996, 789-806. MR1412685 (98g:57023)

[BW2] M. Boileau and S.C. Wang, Degree one maps between small 3-manifolds and Heegaard genus, Algebraic and Geometric Topology 5 (2005), no. 56, 1433-1450. MR2171816 (2006f:57020)

[BoZ] M.Boileau and B. Zimmermann, Symmetries of nonelliptic Montesinos links, Math. Ann. 277, 1987, no. 3, 563-584. MR891592 (88h:57004)

[BuZ] G. Burde and H. Zieschang, Knots, Walter de Gruyter Berlin, 1985 MR808776 (87b:57004)

[CGLS] M. Culler, C. Gordon, J. Luecke, and P. Shalen, Dehn surgery on knots, Ann. of Math. 125, 1987, 237-300. MR881270 (88a:57026)

[Dun1] N. Dunfield, A table of boundary slopes of Montesinos knots, Topology 40, 2001, 309-315. MR1808223 (2001j:57008)

[Dun2] N. Dunfield, Cyclic surgery, degrees of maps of characters curves and volume rigidity for hyperbolic manifolds, Invent. Math. 136, 1999, 623-657. MR1695208 (2000d:57022)

[Ga] D. Gabai, Detecting fibred links in $S^{3}$, Comment. Math. Helv. 61, 1986, 519-555. MR870705 (88c:57009)

[GL] C. Gordon and R. Litherland, Incompressible surfaces in branched coverings, in "The Smith Conjecture" (ed. by H. Bass, J.W. Morgan), Academic Press, 1984, pp. 139-152. MR758466

[Hat] A. Hatcher, On the boundary curves of incompressible surfaces, Pac. J. Math. 99, 1982, 373-377. MR658066 (83h:57016) 
[HT] A. Hatcher and W. Thurston. Incompressible surfaces in 2-bridge knot complements, Invent. Math. 79, 1985, 225-246. MR778125 (86g:57003)

[HWZ] C. Hayat-Legrand, S.C. Wang and H. Zieschang, Minimal Seifert manifolds, Math. Ann. 308, 673-700, 1997. MR1464916 (98i:57029)

[Ka] A. Kawauchi, An imitation theory of manifolds, Osaka J. Math. 26, 1989, 447-464. MR1021425 (90m:57026)

[Ja] W.H. Jaco Lectures on three-manifold topology, AMS, 1977. MR0565450 (81k:57009)

[Mi] J. Milnor, The Schläfli differential equality, in Collected Papers, volume 1, Publish or Perish, 1994. MR1277810 (95c:01043)

[Mo] J.M. Montesinos, Surgery on links and double branched covers of $S^{3}$, in Knots, Groups and Manifolds, Ann. Math. Studies 84, 1975, 227-259. MR0380802 (52:1699)

[O1] U. Oertel, Closed incompressible surfaces in complements of star links, Pac. J. Math. 111, 1984, 209-230. MR732067 (85j:57008)

[O2] U. Oertel, Boundary slopes for Montesinos knots, Topology 28, 1989, 453-480. MR1030987 (91e:57016)

[ORS] T. Ohtsuki, R. Riley and M Sakuma, Epimorphisms between 2-bridge link groups, Manuscript, June 2006.

[RW] R. Reid; S.C. Wang, Non-Haken 3-manifolds are not large with respect to mappings of non-zero degree. Comm. Anal. Geom. 7 1999, no. 1, 105-132. MR1674109 (2000c:57042)

[Ro] D. Rolfsen, Knots and Links, Publish or Perish, Berkeley, 1976. MR0515288 (58:24236)

[Ru] D. Ruberman, Seifert surfaces of knots in $S^{4}$, Pac. J. Math. 145, 1990, 97-116. MR1066400 (91g:57024)

[Th] W. Thurston, Topology and Geometry of 3-manifolds, Princeton Lecture Notes, 1978.

[W] S.C. Wang, Non-zero degree one maps between 3-manifolds, Proceedings of ICM 2002, Vol. II (Beijing, 2002), 457-468, Higher Ed. Press, Beijing, 2002. MR1957056 (2004a:57024)

Laboratoire Émile Picard, CNRS UMR 5580, Université Paul Sabatier, 118 Route de Narbonne, F-31062 Toulouse Cedex 4, France

E-mail address: boileau@picard.ups-tlse.fr

Department of Mathematics, LAMA, Peking University, Beijing 100871, People's RePUBLIC OF CHINA

E-mail address: wangsc@math.pku.edu.cn 